\title{
Cyanide-resistant respiration in diverse marine phytoplankton. Evidence for the widespread occurrence of the alternative oxidase
}

\author{
Niels T. Eriksen ${ }^{1, *}$, Alan J. Lewitus ${ }^{2}$ \\ 'Institute of Biochemistry, Odense University, Campusvej 55, DK-5230 Odense M, Denmark \\ ${ }^{2}$ Belle W. Baruch Institute for Marine Biology and Coastal Research, University of South Carolina, PO Box 1630 , \\ Georgetown, South Carolina 29442, USA
}

\begin{abstract}
In higher plants, the alternative oxidase (AOX) is the terminal oxidase in a mitochondrial electron transport chain thought to allow carbon flow through glycolysis and the citric acid cycle when cellular energy levels are high. In phytoplankton, information is lacking on the taxonomic distribution and metabolic properties of the AOX. We used cyanide-resistant respiration to indicate the presence of the AOX, and the AOX inhibitors, salicylhydroxamic acid (SHAM) and propyl gallate (PG), to estimate the relative activity and capacity of the $\mathrm{AOX}$ in axenic cultures of the marine phytoplankton, Chlorella sp. (Chlorophyceae), Closterium sp. (Zygnematophyceae), Thalassiosira sp. (Bacillariophyceae), Cryptomonas sp. (Cryptophyceae), Ochromonas sp. (Chrysophyceae), and Amphidinium carterae (Dinophyceae), and the freshwater green alga Chlamydomonas reinhardtii. AOX inhibitor effects were higher in stationary phase (nutrient-limited) cultures compared to linearly growing cultures. With the exception of Closterium, in which respiration was almost completely inhibited by cyanide, estimates of AOX capacity, determined as the effect of AOX inhibitors on cyanide-resistant respiration, were nearly identical using the 2 AOX inhibitors, and ranged from 46 to $113 \%$ of the respiration rates of cultures not exposed to inhibitors. The presence of the AOX in 5 of the 6 marine phytoplankton species tested suggests that the AOX is widespread among phytoplankton. Furthermore, the pronounced inhibitory effect of SHAM and PG added alone to stationary phase cultures of Chlorella, Thalassiosira, Cryptomonas, and $A$. carterae ( 21 to $63 \%$ of uninhibited respiration rates) implies that the AOX contributes substantially to oxygen and carbon cycling in many species of phytoplankton during nutrient deficiency.
\end{abstract}

KEY WORDS: Alternative oxidase - Propyl gallate - Salicylhydroxamic acid A Amphidinium Chlamydomonas Chlorella Closterium - Cryptomonas Ochromonas Thalassiosira

\section{INTRODUCTION}

In the mitochondria of higher plants, fungi, protozoa, and some microalgae, electron flow can proceed via 2 respiratory pathways with apparently contrasting physiological functions (reviewed in Mclntosh 1994, Day et al. 1995, Moore et al. 1995, Wagner \& Krab 1995). When electrons pass through the cytochrome pathway (CP), an electrochemical gradient is generated across the inner mitochondrial membrane, and ATP is produced. Alternatively, electrons can be di-

\footnotetext{
•E-mail: nt.eriksen@biochem.ou.dk
}

verted from the $C P$ at the level of ubiquinone, and passed through the alternative oxidase (AOX), which is not linked to production of an electrochemical gradient or ATP. A proposed role of the AOX is to oxidise excess reducing equivalents and to allow glycolytic and citric acid cycle carbon turnover and biosynthetic precursor provision during elevated cellular energy status where high ATP:ADP ratios restrict electron flow through the CP (Day et al. 1995, 1996).

The AOX is resistant to potassium cyanide (KCN) and other $\mathrm{CP}$ inhibitors, but sensitive to several other compounds, among which salicylhydroxamic acid (SHAM) and propyl gallate (PG) have been used to 
quantify activity and capacity of the AOX (Bahr \& Bonner 1973, Moller et al. 1988). Following their convention, AOX activity corresponds to the decrease in respiration rate after addition of $\mathrm{AOX}$ inhibitor, while AOX capacity is estimated as the decrease in respiration rate due to AOX inhibition after the CP has been fully inhibited by e.g. KCN. However, the AOX inhibitors (especially SHAM) may cause side effects such as stimulation of non-mitochondrial peroxidases (Brouwer et al. 1986, Møller \& Bérczi 1986) and nonspecific inhibition of the CP (Møller et al. 1988), and recent evidence indicates that some basic principles justifying the quantification of AOX activity and capacity by inhibitor titrations may be flawed. Estimation of AOX activity is based on the assumption that electrons are not redirected from the AOX to the CP after addition of AOX inhibitor. However, at least in higher plants, inhibition of the AOX may stimulate the CP (Ribas-Carbos et al. 1995, Robinson et al. 1995), with the result that the activity of the AOX is underestimated (Wagner \& Krab 1995, Day et al. 1996). Also, inhibitor-based estimates of AOX capacity may not represent the full capacity, because the activation state of the AOX is regulated by the redox state of the enzyme and the intracellular pyruvate concentration, and the maximum capacity of the AOX therefore may never be attained (Day \& Wiskich 1995, Wagner \& Krab 1995).

Although the inhibitor titrations are likely to underestimate AOX parameters because of the problems above, they are useful for gaining qualitative information on the occurrence of the AOX, and minimal estimates of AOX capacity and activity under defined experimental conditions. Such information is particularly valuable in phytoplankton research, where the AOX has been demonstrated only in a few species of limited taxonomic breadth. Early studies revealed that, in addition to cytochrome oxidase, Chlorella contain a second terminal oxidase resistant to inhibitors like $\mathrm{KCN}$, CO, or $\mathrm{H}_{2} \mathrm{~S}$ (Negelein 1925, Emerson 1927 , Syrett 1951, Sargent \& Taylor 1972). More recent studies on Chlamydomonas reinhardtii have shown that although cyanide-resistant respiration through the AOX may exceed the total rate of respiration of cells not exposed to inhibitors, the addition of AOX inhibitors alone has little or no effect on the respiration rate (Peltier \& Thibault 1985, Goyal \& Tolbert 1989, Weger et al. 1990a, Weger 1996). It therefore has been concluded that the AOX is present but rarely engaged in C. reinhardtii. Cyanide-resistant respiration is especially pronounced during nutrient deficiency (Weger \& Dasgupta 1993), but substantial inhibition of the respiration rate of $C$. reinhardtii by addition of AOX inhibitors alone has been demonstrated only in cultures that have been exposed to different types of stress (high osmotic pressure or temperature), or enriched with glucose or acetate (Weger \& Dasgupta 1993).

The AOX also has been described in heterotrophic Euglena gracilis (Benichou et al. 1988, Devars et al. 1992 Larhrissi et al. 1994) and in the red macroalga Grateloupia doryphora (Robaina et al. 1995), but little is known about the existence and function of the $\mathrm{AOX}$ in other algal taxa. Furthermore, the AOX may not be ubiquitous in phytoplankton, as it appears to be missing in the green alga Selenastrum minutum (Weger et al. 1990b, Lynnes \& Weger 1996). One study, however, suggests that the AOX may be widespread in naturally occurring phytoplankton. Lewitus \& Kana (1995) showed that SHAM, in combination with $\mathrm{KCN}$, decreased respiration rates measured in the light in 4 naturally abundant estuarine phytoplankton species grown mixotrophically on glucose: Pavlova sp. (Prymnesiophyceae), Closterium sp. (Zygnematophyceae), Cryptomonas sp. and Hemiselmis sp. (Cryptophyceae).

We investigated the effects of KCN, SHAM, and PG on the respiration rates of axenic cultures of 6 species of marine phytoplankton; Chlorella sp. (Chlorophyceae), Closterium sp. (Zygnematophyceae), Thalassiosira sp. (Bacillariophyceae), Cryptomonas sp. (Cryptophyceae), Ochromonas sp. (Chrysophyceae), and Amphidinium carterae (Dinophyceae). Our objectives were to test for the presence of the AOX, compare the relative effects of SHAM and PG on total respiration and on cyanide-resistant respiration, and obtain minimal estimates of AOX capacity and activity of light- and nutrient-limited marine phytoplankton belonging to diverse algal taxa. A similar set of experiments with Chlamydomonas reinhardtii (Chlorophyceae) was included to serve as a basis for comparisons.

\section{MATERIALS AND METHODS}

Chlorella sp. HP9101, Closterium sp. HP9101, Thalassiosira sp. HP9101, and Cryptomonas sp. HP9001 were isolated from the Choptank River estuary (a subestuary of Chesapeake Bay) in Maryland, USA. Ochromonas sp. was isolated from the North Inlet estuary in South Carolina, US $\Lambda$, and classified based on pigment composition determined by HPLC analysis (Frigaard et al. 1996). The cultures were made axenic by single-cell isolation by micropipetting under an inverted microscope, except for Chlorella, which was treated with a mixture of $19 \mathrm{mg} \mathrm{l}^{-1}$ penicillin, $30 \mathrm{mg} \mathrm{l}^{l^{-1}}$ streptomycin, and $60 \mathrm{mg}^{-1}$ neomycin (Lewitus \& Kana 1994). Axenic cultures of Amphidinium carterae CCMP1314 and Chlamydomonas reinhardtii UTEX2337 were obtained from the Provasoli-Guillard National Center for Cultivation of Marine Phytoplankton, 
USA, and The Culture Collection of Algae at The University of Texas at Austin, USA, respectively. The cultures were regularly tested for contamination by epifluorescence microscopy, after staining with DAPI (4',6-diamidino-2-phenylindole dihydrochloride hydrate)

All marine species were grown photoautotrophically in batch cultures in $\mathrm{f} / 2$-enriched filtered sea water made with twice the concentrations of nitrate and phosphate described by Guillard (1975). The salinity of the media was adjusted by dilution with distilled water. Amphidinium carterae was grown at a salinity of $30 \%$, and the other marine species at $17 \%$. Chlamydomonas reinhardtii was grown photoautotrophically in the medium described by Starr (1978). All cultures were grown at $25^{\circ} \mathrm{C}$ in continuous light at a photosynthetic photon flux density of 100 to $130 \mu \mathrm{mol} \mathrm{m}^{-2} \mathrm{~s}^{-1}$, measured by a Biospherical Instruments Quantum Scalar Irradiance Meter QSL-100 inside growth flasks containing sterile medium.

Respiration experiments were performed on linearly growing (light-limited) or stationary phase (nutrientlimited) cultures. Immediately before measuring respiration, 20 to $100 \mathrm{ml}$ of culture was centrifuged for $10 \mathrm{~min}$ in a Sorvall Ultracentrifuge at 3000 to $7000 \times \mathrm{g}$ (depending on species), and resuspended in $2 \mathrm{ml}$ of growth medium. An aliquot of the concentrated cell suspension was transferred to a $1 \mathrm{ml}$ closed respiration cell (Strathkelvin Instruments RC 350) equipped with an oxygen micro-electrode (Strathkelvin Instruments 1302 microcathode oxygen electrodej. The construction of the measuring cell excluded air bubbles and allowed addition of inhibitors to stirred cultures during the measurements. Prior to the inhibitor additions, the respiration rates of the cell suspensions (determined in the dark from the decrease in dissolved oxygen concentration) were between 2 and $20 \mu \mathrm{M} \mathrm{O}_{2} \mathrm{~min}^{-1}$.

Inhibitors were added ( 0.5 to $10 \mu l$ per addition) to the measuring cell from stock solutions of $100 \mathrm{mM}$ SHAM and $10 \mathrm{mM} \mathrm{PG}$ in 2-methoxyethanol, and $20 \mathrm{mM} \mathrm{KCN}$ in water. Additions of similar amounts of 2-methoxyethanol alone did not affect the respiration rate of the algae.

\section{RESULTS}

The respiration rate of Closterium was almost completely inhibited by $\mathrm{KCN}$ (Fig. 1), indicating little or no AOX capacity in this species. In Cryptomonas, respiration was only partly inhibited, and in Thalassiosira, Ochromonas, Amphidinium carterae, and Chlorella, the respiration rate was stimulated by KCN. Stimulation of respiration by $\mathrm{KCN}$ is observed in cultures where the AOX capacity exceeds the $\mathrm{CP}$ activity, and

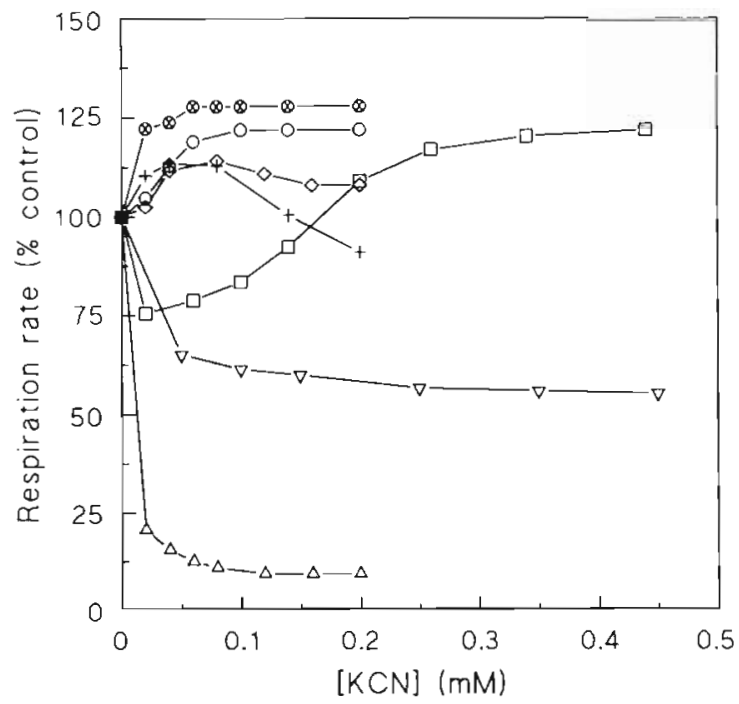

Fig. 1. Respiration rates in presence of $\mathrm{KCN}$ of Chlamy. domonas reinhardtii (o), Chlorella $\mathrm{sp}$. (), Closterium sp. $(\Delta)$, Thalassiosira sp. (o), Cryptomonas sp. ( $\nabla)$, Ochromonas sp $(\otimes)$, and Amphidinium carterae (+) from stationary phase cultures. Each point is the average of 2 to 4 measurements performed on cells from the same cultures as in Figs. 2 \& 3 . Rates are the percentage of total respiration rates of uninhibited controls

has been explained as a 'Pasteur' effect resulting from reduced cellular ATP levels (Weger \& Dasgupta 1993). In titration experiments using $\mathrm{AOX}$ inhibitors in combination with KCN (Figs. 2 \& 3), KCN was added at the lowest concentration that gave maximal inhibition (0.25 $\mathrm{mM}$ in Cryptomonas) or stimulation $(0.1 \mathrm{mM}$ in Thalassiosira, Ochromonas, and A. carterae; $0.25 \mathrm{mM}$ in Chlorella) of the respiration rate. At these concentrations, the CP was considered to be completely inhibited, and other effects of KCN kept at a minimum.

Figs. $2 \& 3$ show the respiratory responses of the phytoplankton to increasing concentrations of SHAM and $P G$, with and without prior addition of $K C N$. All measurements on each species were performed on cells from the same culture harvested during stationary growth phase, because preliminary trials showed higher effects of AOX inhibitors in stationary phase cultures than in cultures in the growth phase. The SHAM- or PG-induced decrease in cyanide-resistant respiration rate in the presence of $\mathrm{KCN}$ is designated $V_{\text {alt }}$ (minimal estimate of AOX capacity) and the AOX inhibitor-induced decrease in respiration rate in the absence of $\mathrm{KCN}$ is designated $\mathrm{v}_{\text {alt }}$ (minimal estimate of AOX activity).

With the exception of Closterium, in which $\mathrm{KCN}$ caused more than a $90 \%$ decrease in respiration rate, $V_{\text {alt }}$ measurements were between 46 and $113 \%$ of the respiration rates of uninhibited cultures, suggesting that the AOX is present in relatively high amounts in 5 


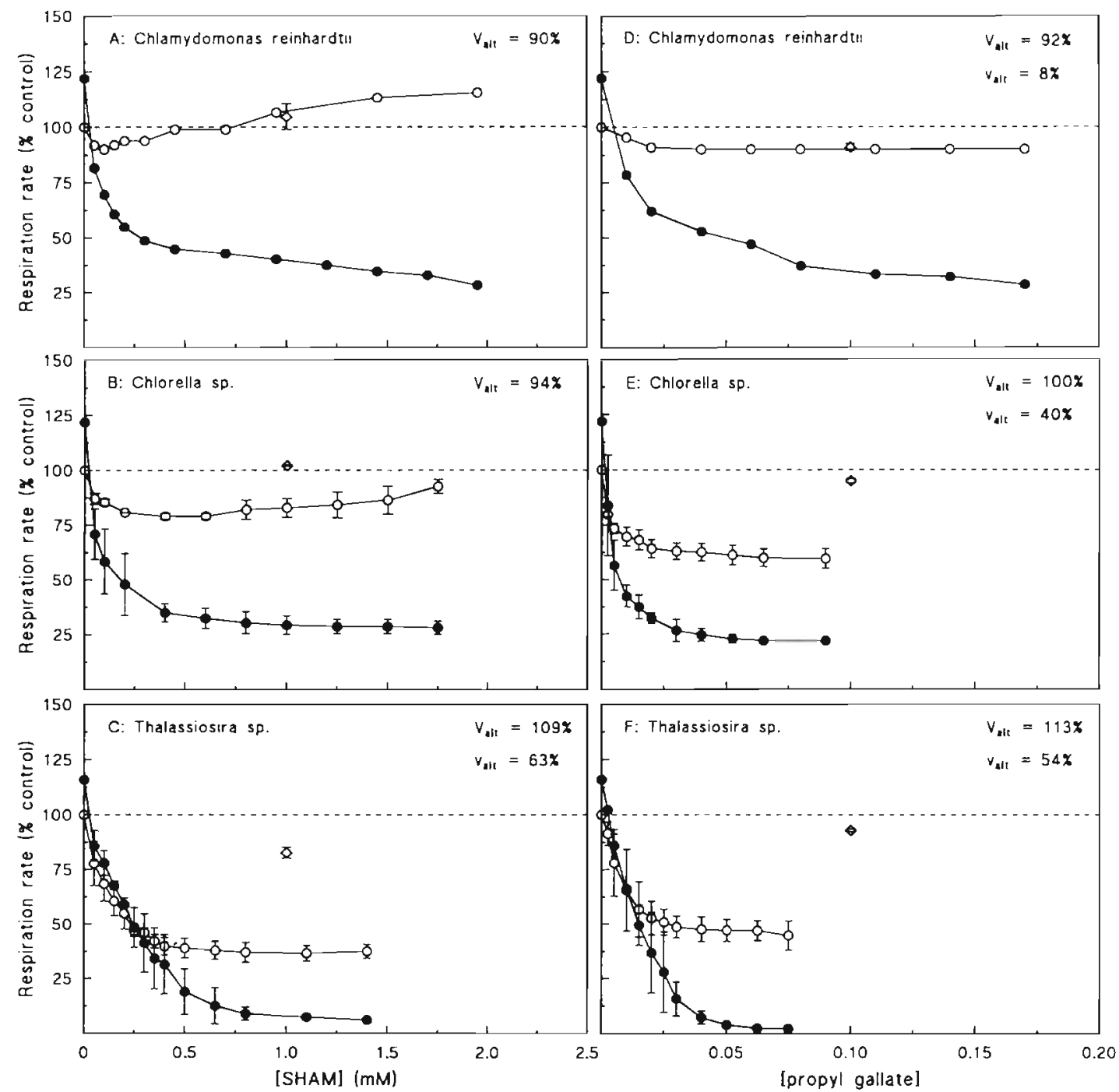

Fig. 2. Respiration rates in presence of SHAM (A-C) and PG (D-F) added alone (O) and in combination with KCN (•) of Chlamydomonas reinhardtii, Chlorella sp., and Thalassiosira sp. from stationary phase cultures. Respiration rates of linearly growing cultures in presence of $1 \mathrm{mM}$ SHAM and $0.1 \mathrm{mM}$ PG are also shown ( $\diamond)$. Rates are the percentage of total respiration rates of uninhibited controls, indicated by broken line. $V_{\text {alt }}$ and $v_{\text {alt }}$ indicate the percent decrease in respiration rate caused by $A O X$ inhibitor addition to cultures previously inhibited by $\mathrm{KCN}$ and cultures not exposed to $\mathrm{KCN}$, respectively

of the 6 marine species (Figs. $2 \& 3$ ). In fact, in 4 of those species and in Chlamydomonas reinhardtii, estimates of $V_{\text {alt }}$ reached $90 \%$ and above. In all species, these estimates varied only slightly with AOX inhibitor type (SHAM and PG responses differed by $14 \%$ in Amphidinium carterae and less than $6 \%$ in the other species).

Stimulation of respiration by SHAM in Chlamydomonas reinhardtii and Chlorella (Fig. 2A, B) and by PG in Amphidinium carterae (Fig. 3F) prevented proper estimates of $v_{\text {alt }}$ from these experiments. In the other experiments, the effects of SHAM and PG ranged from only a slight reduction of the respiration rates $(8 \%$ in C. reinhardtii and Ochromonas exposed to
PG) to more pronounced inhibitions of 21 to $63 \%$ in the other species. Also, with the exception of C. reinhardtii and Ochromonas, the effect of AOX inhibitor addition. on cells from the stationary growth phase were consistently higher than on cells from linearly growing cultures. Respiration rates of cells from linearly growing cultures exposed to $1 \mathrm{mM}$ SHAM or $0.1 \mathrm{mM} \mathrm{PG}$ are included in Figs. 2 \& 3.

The effects of relatively high concentrations of SHAM and PG on cultures not exposed to $\mathrm{KCN}$ were examined in titration experiments conducted on linearly growing cultures where effects from AOX inhibition presumably are at a minimum (Fig. 4). SHAM concentrations below $5 \mathrm{mM}$ stimulated respiration in Chlamydomonas rein- 

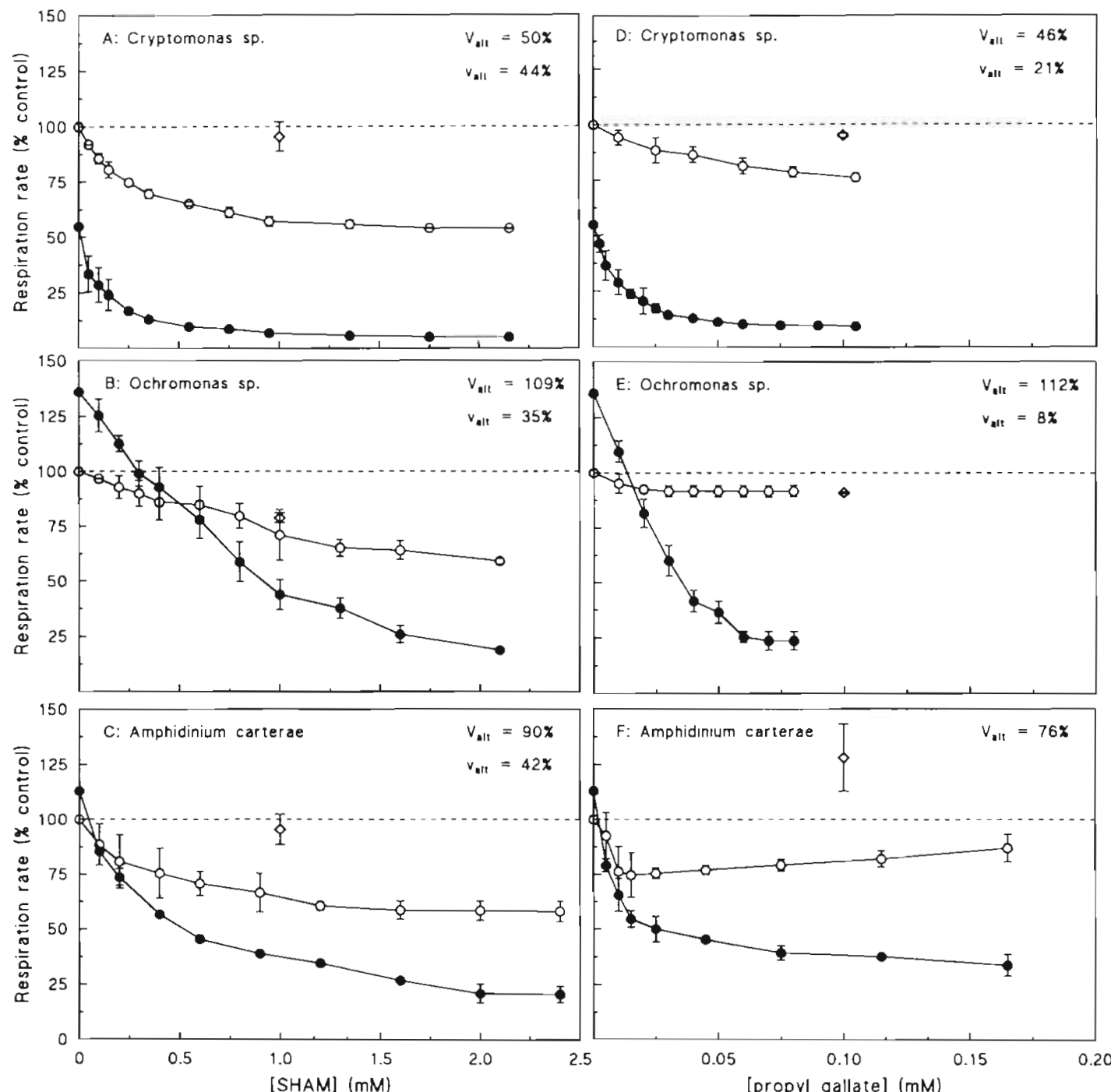

Fig. 3. Respiration rates in presence of SHAM (A-C) and PG (D-F) added alone (O) and in combination with KCN ( $\bullet$ ) of Cryptomonas sp., Ochromonas sp., and Amphidinium carterae from stationary phase cultures. Respiration rates of linearly growing cultures in presence of $1 \mathrm{mM}$ SHAM and $0.1 \mathrm{mM} \mathrm{PG}$ are also shown (o). Rates are the percentage of total respiration rates of uninhibited controls, indicated by broken line. $V_{\text {alt }}$ and $v_{\text {alt }}$ indicate the percent decrease in respiration rate caused by $A O X$ inhibitor addition to cultures previously inhibited by $\mathrm{KCN}$ and cultures not exposed to $\mathrm{KCN}$, respectively

hardtii, Chlorella, and Amphidinium carterae (Fig. 4A), possibly resulting from stimulation of extramitochondrial peroxidases, while SHAM concentrations above $5 \mathrm{mM}$ were inhibitory to all species. PG slightly stimulated respiration in Chlorella, Closterium, and Cryptomonas at concentrations above 0.5 or $1 \mathrm{mM}$, but strongly stimulated respiration at much lower concentrations in A. carterae (Fig. 4B).

\section{DISCUSSION}

The phytoplankton species used here showed various degrees of insensitivity to KCN and sensitivity to SHAM and PG, when these inhibitors were added to cultures not exposed to KCN. Closterium was the only species in which respiration was not partly KCN-resistant. Some of the different responses to SHAM and PG by the other species are indicative of side effects that have been attributed to these inhibitors. SHAM-induced stimulation of oxygen uptake by extramitochondrial peroxidases may have occurred in Chlamydomonas reinhardtii, Chlorella, and Amphidinium carterae (Fig, 4A), as has been described in higher piants (Brouwer et al. 1986, Møller \& Bérczi 1986) and in green algae (Torkelson et al. 1995, Weger et al. 1996)

Non-specific inhibition at SHAM concentrations above $5 \mathrm{mM}$ was observed in all species (Fig. 4A). 

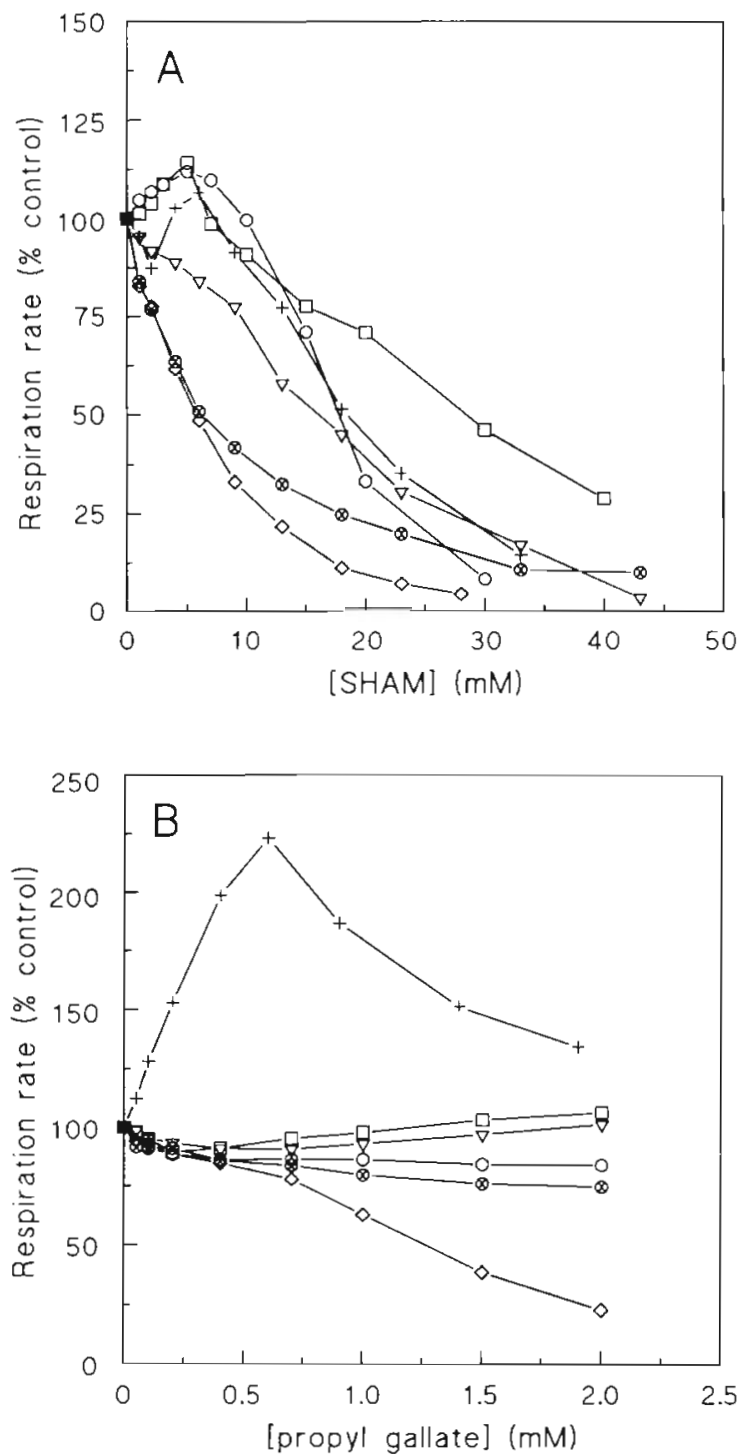

Fig. 4. Respiration rates in presence of relatively high concentrations of SHAM (A) and PG (B) on respiration rates of Chlamydomonas reinhardtii (O), Chlorella sp. (), Thalassiosira sp. ( (), Cryptomonas sp. ( $\nabla)$, Ochromonas sp. $(\otimes)$, and Amphidinium carterae (+). Each point is the average of 2 to 3 measurements performed on cells from linearly growing batch cultures. Rates are the percentage of total respiration. rates of uninhibited controls

SHAM concentrations above $2 \mathrm{mM}$ are known to inhibit the CP (Møller et al. 1988). Therefore, the plateaus in respiration rates observed at 1 to $2 \mathrm{mM}$ SHAM in Figs. $2 \mathrm{C} \& 3 \mathrm{~A}-\mathrm{D}$, where the AOX is fully inhibited and the $\mathrm{CP}$ thought to be unaffected, are maintained only within very narrow concentration intervals.

Inhibition of respiration by PG is thought to be more specific to the AOX than the more commonly used SHAM (Weger \& Dasgupta 1993, Hoefnagel et al. 1995, Robinson et al. 1995), and substantial side effects caused by PG were observed only in Amphidinium carterae (Fig. 4B). In comparison to SHAM, PG has the reputed disadvantage of relatively poor penetrability (Robinson et al. 1995). However, our results show that algal cells are permeable to $\mathrm{PG}$, as maximal inhibition was obtained 2 to 3 min after PG additions (consistent with the findings of Weger \& Dasgupta 1993).

The respiration rate of Chlamydomonas reinhardtii in the stationary or linear growth phase was stimulated by SHAM and only slightly inhibited ( $8 \%$ ) by PG when added to cultures not exposed to $\mathrm{KCN}$. This is consistent with previous reports that, in both nutrient-sufficient and nutrient-deficient $C$. reinhardtii, the respiration rate is, at most, only slightly affected by SHAM or PG (Peltier \& Thibault 1985, Goyal \& Tolbert 1989, Weger et al. 1990a, Weger 1996). In contrast to C. reinhardtii, however, the respiration rates of stationary phase cultures of Chlorella, Thalassiosira, Cryptomonas, and Amphidinium carterae were substantially decreased (by 21 to $63 \%$ ) by SHAM or PG when added alone (Figs. $2 \& 3$ ), suggesting that at least a similar proportion of the total respiration rate was carried out by the AOX. Though $C$. reinhardtii has been the research subject in almost all recent studies on the AOX in phytoplankton, the difference in AOX inhibitor response between C. reinhardtii and other species used in our experiments suggests caution in extrapolating AOX properties in C. reinhardtii to those of other phytoplankton species.

With the exceptions of Chlamydomonas reinhardtif and Ochromonas, AOX inhibitor addition had a relatively greater effect on cultures in stationary growth phase than on those in linear growth phase (Figs. 2 \& $3)$. These results suggest that a substantial proportion of the AOX may be engaged in nutrient-depleted phytoplankton, consistent with the proposed role of the AOX as a stress response to nutrient deficiency in higher plants (Rychter \& Mikulska 1990, Rychter et al. 1992, Hoefnagel et al. 1993). However, it should be emphasized that our experiments do not reveal whether the relatively lower inhibition by SHAM or PG in linearly growing cultures resulted from lower AOX activity or from the diversion of electrons from the AOX to the CP upon inhibitor addition (Ribas-Carbos et al. 1995, Robinson et al. 1995)

In all species except Closterium, pronounced minimal estimates of AOX capacity were obtained, reaching $90 \%$ or more in 5 species. Respiratory inhibitor titrations can provide only indirect evidence for the presence of the AOX. Direct confirmation of the AOX based on a cross-reaction between cell extracts and an antibody raised against the AOX from voodoo lily, Sauromatum guttatum (Elthon et al. 1989), has been reported in Chlamydomonas reinhardtii (Derzaph \& Weger 1996). Also, several studies have demonstrated 
correlations between AOX inhibitor effects on cyanide-resistant respiration and AOX content in higher plants (Hiser \& McIntosh 1990, Obenland et al. 1990, Stewart et al. 1990, Rhoads \& McIntosh 1992, Vanler berghe \& McIntosh 1992). Moreover, the nearly identical $V_{a l t}$ estimates from SHAM and PG titration experiments in our study provides further support that the effect of these inhibitors on cyanide-resistant respiration reflects the capacity of the AOX under defined experimental conditions.

The presence of the AOX has been reported in some members of Euglenophyceae, Chlorophyceae, and Rhodophyceae. This study suggests that the AOX also may be present in members of Bacillariophyceae Cryptophyceae, Chrysophyceae, and Dinophyceae. Although inhibitor titrations may provide underestimates of AOX activity and capacity, the general findings of pronounced AOX inhibitor effects on stationary phase cultures with or without prior inhibition of the $\mathrm{CP}$ by $\mathrm{KCN}$ suggest that the AOX is widespread among phytoplankton and plays a significant role in their metabolism during nutrient deficiency.

Acknowledgements. The research was conducted at the University of South Carolina's Baruch Marine Laboratory in Georgetown, South Carolina, USA. We thank Bonnie Willis and Chris Burbage for technical assistance, and Raymond P. Cox for conducting the pigment analysis. N.T.E. was supported by The Plasmid Foundation and Odense University Research Foundation. The research also was funded by N.S.F. Grants OCE-9315663 and DEB-9509057, and NOAA Grant NA90AA-D-SG672 to A.J.L. This is contribution no. 1178 of the Belle W. Baruch Institute of Marine Biology and Coastal Research.

\section{LITERATURE CITED}

Bahr JT, Bonner W1) (1973) Cyanide-insensitive respiration 1. The steady states of skunk cabbage spadix and bean hypocotyl mitochondria. J Biol Chem 248:3441-3445

Benichou P, Calvayrac R, Claisse M (1988) Induction by antimycin $\mathrm{A}$ of cyanide-resistant respiration in heterotrophic Euglena gracilis: effects on growth, respiration and protein biosynthesis. Planta 175:23-32

Brouwer SK, van Valen T, Day DA, Lambers H (1986) Hydroxamate-stimulated $\mathrm{O}_{2}$ uptake in roots of Pisum sativun and Zea mays, mediated by a peroxidase. Its consequences for respiration measurements. Plant Physiol 82:236-240

Day DA, Whelan J, Millar AH, Siedow JN, Wiskich JT (1995) Regulation of the alternative oxidase in plants and fungi. Aust J Plant Physiol 22:497-509

Day DA, Krab K, Lambers H, Moore AL, Siedow JN, Wagner AM, Wiskich JT (1996) The cyanide-resistant oxidase: to inhibit or not to inhibit, that is the question. Plant Physiol 110:1-2

Day DA, Wiskich JT (1995) Regulation of alternative oxidase activity in higher plants. J Bioenerg Biomembr 27:379-385

Derzaph TLM, Weger HG (1996) Immunological identification of the alternative oxidase in Chlamydomonas reinhardti (Chlorophyta). J Phycol 32:621-623
Devars S, Torres-Márquez ME, González-Halphen D, Uribe A, Moreno-Sánchez R (1992) Cyanide-sensitive and cyanide-resistant respiration of dark-grown Euglena gracilis. Plant Science 82:37-46

Elthon TE, Nickels RL, McIntosh L (1989) Monoclonal antibodies to the alternative oxidase of higher plant mitochondria. Plant Physiol 89:1311-1317

Emerson R (1927) The effect of certain respiratory inhibitors on the respiration of Chlorella. J Gen Physiol 10:469-477

Frigaard NU, Larsen KL، Cox RP (1996) Spectrochromatography of photosynthetic pigments as a fingerprinting technique for microbial phototrophs. FEMS Microbiol Ecol 20:69-77

Goyal A. Tolbert NE (1989) Variations in the alternative oxidase in Chlamydomonas grown in air or high $\mathrm{CO}_{2}$. Plant Physiol 89:958-962

Guillard RRL (1975) Culture of phytoplankton for feeding marine invertebrates. In: Smith WL, Chanley MH (eds) Culture of marine invertebrates. Plenum Press, New York, p 29-60

Hiser C, Mclntosh L (1990) Alternative oxidase of potato is an integral membrane protein synthesized de novo during aging of tuber slices. Plant Physiol 93:312-318

Hoefnagel MHN, van Iren F, Libbenga KR (1993) In suspension cultures of Catharanthus roseus the cyanide-resistant pathway is engaged in respiration by excess sugar in combination with phosphate or nitrogen starvation. Physiol Plant 87:297-304

Hoefnagel MHN, Wiskich. JT, Madgwick SA, Patterson Z, Oettmeier $W$, Rich $R$ (1995) New inhibitors of the ubiquinol oxidase of higher plant mitochondria. Eur J Biochem 233:531-537

Larhrissi Z, Bomsel JL, Calvayrac R (1994) Relative effect of 2,4-dinitrophenol on the respiratory chain of heterotrophic Euglena gracilis adapted and non-adapted to antimycin A. J Plant Physiol 143:264-268

Lewitus AJ, Kana TM (1994) Responses of estuarine phytoplankton to exogenous glucose: stimulation versus inhibition of photosynthesis and respiration. Limnol Oceanogr 39:182-189

Lewitus AJ, Kana TM (1995) Light respiration in six estuarine phytoplankton species: contrasts under photoautotrophic and mixotrophic growth conditions. J Phycol 31:754-761

Lynnes JA, Weger HG (1996) Azide-stimulated oxygen consumption by the green alga Selenastrum minutum. Physiol Plant 97:132-138

McIntosh L (1994) Molecular biology of the alternative oxidase. Plant Physiol 105:781-786

Moore AL, Umbach AL, Siedow JN (1995) Structure-function relationships of the alternative oxidase of plant mitochondria: a model of the active site. J Bioenerg Biomembr 27 $367-377$

Møller IM, Bérczi A (1986) Salicylhydroxamic acid-stimulated NADH oxidation by purified plasmalemma vesicles from wheat roots. Physiol Plant 68:67-74

Møller IM, Bérczi A, van der Plas LHW, Lambers H (1988) Measurement of the activity and capacity of the alternative pathway in intact plant tissues: identification of problems and possible solutions. Physiol Plant 72:642-649

Negelein E (1925) Über die Wirkung des Schwefelwasserstoffs auf chemische Vorgänge in Zellen. Biochem Z 165: 203-213

Obenland D. Diethelm R, Shibles R, Stewart C (1990) Relationship of alternative respiratory capacity and alternative oxidase amount during sotbean seedling growth. Plant Cell Physiol 31:897-901

Peltier $\mathrm{G}$, Thibault $\mathrm{P}$ (1985) $\mathrm{O}_{2}$ uptake in the light in Chlamy domonas. Evidence for persistent mitochondrial respira- 
tion. Plant Physiol 79:225-230

Rhoads DM, McIntosh L (1992) Salicylic acid regulation of respiration in higher plants: alternative oxidase expression. Plant Cell 4:1131-1139

Ribas-Carbo M, Berry JA, Yakir D, Giles L, Robinson SA, Lennon AM, Siedow JN (1995) Electron partitioning between the cytochrome and alternative pathways in plant mitochondria. Plant Physiol 109:829-837

Robaina RR, Garciajimenez P, Brito I, Luque A (1995) Light control of the respiration of exogenous glycerol in the red macroalga Grateloupia doryphora. Eur J Phycol 30: $81-86$

Robinson SA, Ribas-Carbo M, Yakir D, Giles L, Reuveni Y, Berry JA (1995) Beyond SHAM and cyanide: opportunities for studying the alternative oxidase in plant respiration using oxygen isotope discrimination. Aust J Plant Physiol 22:487-496

Rychter AM, Mikulska M (1990) The relationship between phosphate status and cyanide-resistant respiration in bean roots. Physiol Plant 79:663-667

Rychter AM, Chauveau M, Bomsel JL, Lance C (1992) The effect of phosphate deficiency on mitochondrial activity and adenylate levels in bean roots. Physiol Plant 84: $80-86$

Sargent DF, Taylor CPS (1972) Terminal oxidases of Chlorella pyrenoidosa. Plant Physiol 49:775-778

Starr RC (1978) The culture collection of algae at the University of Texas at Austin. J Phycol 14:47-100

Stewart CR, Martin BA, Reding L, Cerwick S (1990) Respiration and alternative oxidase in corn seedling tissues dur-

Editorial responsibility: William Li,

Dartmouth, Nova Scotia, Canada ing germination at different temperatures. Plant Physiol $92: 755-760$

Syrett PJ (1951) The effect of cyanide on respiration and the oxidative assimilation of glucose by Chlorella vulgaris. Ann Bot 15:473-492

Torkelson JD, Lynnes JA, Weger HG (1995) Extracellular peroxidase-mediated oxygen consumption in Chlamydomonas reinhardtii (Chlorophyta). J Phycol 31:562-567

Vanlerberghe GC, McIntosh L (1992) Coordinate regulation of cytochrome and alternative pathway respiration in tobacco. Plant Physiol 100:1846-1851

Wagner AM, Krab K (1995) The alternative respiration pathway in plants: role and regulation. Physiol Plant 95:318-125

Weger HG (1996) Interactions between respiration and inorganic phosphate uptake in phosphate-limited cells of Chlamydomonas reinhardti. Physiol Plant 97:635-642

Weger HG, Dasgupta R (1993) Regulation of alternative pathway respiration in Chlamydomonas reinhardtii (Chlorophyceae). J Phycol 29:300-308

Weger HG, Chadderton AR, Lin M, Guy RD, Turpin DH (1990a) Cytochrome and alternative pathway respiration during transient ammonium assimilation by $\mathrm{N}$-limited Chlamydomonas reinhardtii. Plant Physiol 94:1131-1136

Weger HG, Guy RD, Turpin DH (1990b) Cytochrome and alternative pathway respiration in green algae. Measurements using inhibitors and ${ }^{18} \mathrm{O}_{2}$ discrimination. Plant Physiol 93:356-360

Weger HG, Lynnes JA, Torkelson JD (1996) Characterization of extracellular oxygen consumption by the green alga Selenastrum minutum. Physiol Plant 96:268-274

Submitted: June 2, 1998; Accepted: September 25, 1998

Proofs received from author(s): April 28, 1999 